\title{
Evidence for a calorically costly negative consummatory contrast effect in rats
}

\author{
FRED P. VALLE \\ University of British Columbia, Vancouver, British Columbia, Canada
}

\begin{abstract}
For $2 \mathrm{~h}$ prior to their daily meal of Purina Chow, rats (which were $14 \%$ below ad-lib weight levels) had access to a sucrose solution. For half (Group 16-4), the solution was alternated daily between $16 \%$ and $4 \%$; for the other half (Group 4-4), the solution was always $4 \%$. On $16 \%$ days, Group 16-4 consumed significantly more calories and gained significantly more weight than did Group 4-4, because of a greater consumption of sucrose calories (Purina intake was similar for the two groups). On 4\% days, however, Group 16-4 consumed both significantly fewer sucrose calories and significantly fewer Purina calories than did Group 4-4. These two contrast effects resulted in a $17 \%$ shortfall in total caloric intake for Group 16-4 on 4\% days. As a consequence, Group 16-4 showed a significant drop in body weight, compared to Group 4-4, on $4 \%$ days. A second experiment was carried out to investigate whether the contrast-induced reduction in Purina intake shown by Group $16-4$ on $4 \%$ days would be eliminated if (1) a 30 -min interval separated sucrose ingestion from Purina ingestion, or (2) a 25-min interval plus 5-min exposure to $16 \%$ sucrose separated ingestion of $4 \%$ sucrose from Purina ingestion. Purina intake was still suppressed in Group 16-4 under both conditions.
\end{abstract}

A negative consummatory contrast effect (NCCE) is said to occur when animals that have experience with both a more and a less preferred incentive consume less of the latter than do animals that have experience only with the less preferred substance (see Flaherty, 1982, for a general review of contrast effects). Such contrast effects have proven to be of interest, because their occurrence demonstrates that an individual's responsiveness to a current incentive is determined, not only by the absolute properties of that substance as a stimulus, but also by the individual's experience with other incentives ingested in the same situation, and by the hedonic properties of those other incentives relative to those of the current incentive. These findings have stimulated theoretical attempts to conceptualize the psychological processes or mechanisms underlying such effects (see, e.g., Bolles, 1975).

From a biopsychological perspective, an NCCE could be viewed as a maladaptive behavioral trait. That is to say, a hungry organism that consumes less of a nutritious food than time permits, simply because that food is not as attractive as some other food, currently unavailable, that has been encountered in the past, is foregoing needed calories, unnecessarily. However, in the studies in which NCCEs have been demonstrated, the subjects have been

\footnotetext{
I would like to thank Colin McEown, who assisted in data collection, and whose work was funded by an Opportunities ' 89 summer grant from The Province of British Columbia. I would also like to thank A. T. Phillips, who generously supplied the animals for the study, and two anonymous reviewers, whose comments were very helpful during my revision of the manuscript. Correspondence should be addressed to Fred P. Valle, Department of Psychology, University of British Columbia, Vancouver, British Columbia V6T 1Y7, Canada.
}

exposed to the substances in question for only a few minutes each day. Such procedures render the caloric consequences of NCCEs negligible, and hence leave open the question of whether an NCCE could be of sufficient magnitude as to be maladaptive. An example of a typical study might be helpful.

Flaherty, Becker, and Pohorecky (1985) exposed one group of hungry rats to a $32 \%$ sucrose solution for $5 \mathrm{~min}$ a day for 10 days, and then, on the following day, to a $4 \%$ sucrose solution for $5 \mathrm{~min}$. A group of control animals was exposed to the $4 \%$ solution on all days. On the 11 th day, the shifted group licked the $4 \%$ solution approximately 400 times, whereas the control group licked the same solution approximately 1,200 times. While the behavioral difference is striking, the caloric consequences of that difference are not. If it is assumed that each lick at a standard drinking tube produces approximately $.005 \mathrm{cc}$ of solution (Bolles, 1975, p. 203), then the control animals ingested about $6 \mathrm{cc}$ of the solution, while the shifted animals ingested about $2 \mathrm{cc}$. Six centiliter of a $4 \%$ sucrose solution yield about $0.9 \mathrm{kcal}$, while $2 \mathrm{cc}$ yield about $0.3 \mathrm{kcal}$. Thus, the NCCE "cost" the shifted animals $0.6 \mathrm{kcal}$. But, given that the animals were probably eating at least $40-50 \mathrm{kcal}$ following each meal (the size of the daily ration is not reported; the animals were fed enough to maintain them at $82 \%$ ad-lib body weight), $0.6 \mathrm{kcal}$ does not represent a sizeable fraction of total daily caloric intake.

The present experiments, however, present evidence of contrast effects in rats that cost the animals approximately $17 \%$ of their potential caloric intake on each of several days, resulting in significant and unnecessary reductions in body weight. These body-weight losses oc- 
curred in animals that were already substantially below their ad-lib weight levels.

\section{EXPERIMENT 1}

\section{Method}

Subjects. The subjects were 14 male rats of the Charles River strain. The animals were experimentally naive and approximately 200 days old; they weighed an average of $568 \mathrm{~g}$ at the start of the experiment. They were housed in standard individual metal cages in a colony room maintained at $21^{\circ}$, under a 12:12-h light:dark cycle, with light onset occurring at $0700 \mathrm{~h}$.

Procedure. On Day 0, the food (Purina Chow) was removed from all animals at $1600 \mathrm{~h}$. On Days 1-9, the animals were weighed (to the nearest gram) at $1400 \mathrm{~h}$ and were then given access to Purina Chow for $2 \mathrm{~h}$. Water was available ad lib. Food and water intake were determined by weight (to the nearest $0.1 \mathrm{~g}$ ) each day. On Day 10, the same procedure was followed, except that the water was removed following the meal, to ensure that the animals would be thirsty and inclined to sample a sucrose solution when it was introduced the following day. This was the only occasion on which the animals did not have access to water following meals.

On Day 11 , the animals were weighed at $1200 \mathrm{~h}$ and were then given access to a sucrose solution for $2 \mathrm{~h}$. At $1400 \mathrm{~h}$, the sucrose was removed and weighed (to the nearest $0.1 \mathrm{~g}$ ), and Purina Chow and water were provided. At $1600 \mathrm{~h}$, the food was removed and weighed; the water was left on the cages until $1200 \mathrm{~h}$ the following day. On the next day, and thereafter until Day 30, the animals were weighed and the water was removed at $1200 \mathrm{~h}$, and a sucrose solution was provided for $2 \mathrm{~h}$. This was followed by 2 -h access to Purina Chow and water, with the water remaining available until $1200 \mathrm{~h}$ the next day. A final weighing of the animals took place at $1200 \mathrm{~h}$ on Day 31 . In summary: For 20 days, beginning on Day 11, the animals had (1) 2-h access to a sucrose solution, followed by (2) 2 -h access to Purina Chow and water, followed by (3) 20-h access to water.

For 7 of the animals, chosen randomly, the sucrose solution was alternated daily between $16 \%$ and $4 \%$, beginning with $16 \%$ on Day 11. These experimental animals are hereafter designated Group 16-4. The remaining 7 control animals (Group 4-4) always received a $4 \%$ solution. Fresh solutions (weight/volume) were prepared every other day and kept at room temperature.

\section{Results and Discussion}

Body weight on Day 11. On Day 11, when exposure to sucrose began, the animals weighed an average of $488 \mathrm{~g}$, or $86 \%$ of their weight on Day 0 . Hence, compared with their weight under ad-lib conditions, the animals were in caloric deficit when given the opportunity to ingest sucrose calories.

Caloric intake. Figure 1 shows the average total daily caloric intake (in kilocalories) of the two groups (1) on odd days, when Group $16-4$ had access to $16 \%$ and Group 4-4 had access to $4 \%$ sucrose, and (2) on even days, when both groups had access to $4 \%$ sucrose. Caloric intake for any given substance was determined by multiplying the amount (in grams) ingested by the number of kilocalories per gram: 3.61 for Purina Chow, and .15 and .58 for the $4 \%$ and $16 \%$ sucrose solutions, respectively. (Pure sucrose yields $3.85 \mathrm{kcal}$ per gram; thus, the $4 \%$ solution contained $54 \mathrm{kcal}$ per $1,016 \mathrm{~g}$ [i.e., per liter], while the $16 \%$ solution contained $616 \mathrm{kcal}$ per $1,062 \mathrm{~g}$ [i.e., per liter].)

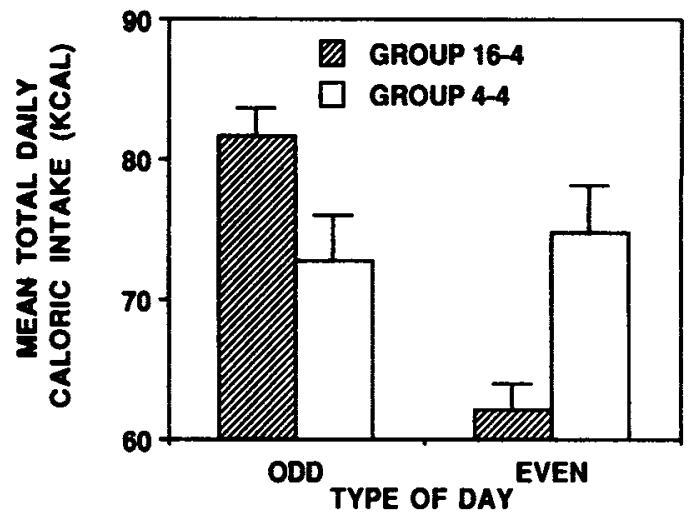

Figure 1. Mean total (sucrose + Purina) daily caloric intake (in kilocalories) in Experiment 1, as a function of the concentration of sucrose available for $2 \mathrm{~h}$ prior to a 2 -h Purina meal. For Group 16-4, the solution was $16 \%$ sucrose on odd days, and $4 \%$ sucrose on even days; for Group $4-4$, the solution was always $4 \%$. Vertical lines indicate 1 SEM.

On odd days, Group 16-4 consumed significantly more total calories than did Group 4-4 $[t(12)=3.16$, $p<.01]$. This difference was due entirely to sucrose intake: Group 16-4 consumed an average of 20.6 sucrose kcal per day, while Group 4-4 consumed an average of 10.3 sucrose kcal per day $[t(12)=7.21, p<.001]$. The two groups consumed similar numbers of Purina calories on odd days: 60.9 and $62.4 \mathrm{kcal}$ for Groups $16-4$ and 4-4, respectively $[t(12)=0.44]$.

On even days, however, when both groups had access to the $4 \%$ solution, Group $16-4$ consumed significantly fewer total calories than did Group $4-4[t(12)=3.16$, $p<.01]$. This difference in total caloric intake reflected both a significant difference in the number of sucrose calories consumed, with Group 16-4 consuming a daily average of $7.1 \mathrm{kcal}$ and Group 4-4 an average of $11.0 \mathrm{kcal}$ $[t(12)=3.47, p<.01]$, and a significant difference in the number of Purina calories consumed each day, with Group 16-4 consuming an average of $55.1 \mathrm{kcal}$ and Group $4-4$ an average of $63.7 \mathrm{kcal}[t(12)=2.36$, $p<.05]$.

Figure 2 shows the average daily intake of sucrose kilocalories for the two groups on even (4\%) days, over 2 -day blocks of even days. It can be seen that the size of the negative contrast effect increased substantially over days. A mixed ANOVA, with groups as a betweensubjects variable and blocks as a within-subjects variable, revealed a significant groups effect, reflecting the overall greater sucrose intake of Group 4-4 that has already been described $[F(1,12)=12.04, p<.01]$; a significant blocks effect, reflecting an overall increase in sucrose consumption over days $[F(4,48)=11.26, p<.001]$; and a significant interaction between groups and blocks, reflecting the much greater increase over days for Group 4-4, compared to Group $16-4[F(4,48)=5.32, p<.05]$.

Figure 3 shows the average daily intake of Purina kilocalories for the two groups on even (4\%) days, over 2-day blocks of even days. In this instance, as can be seen, the 


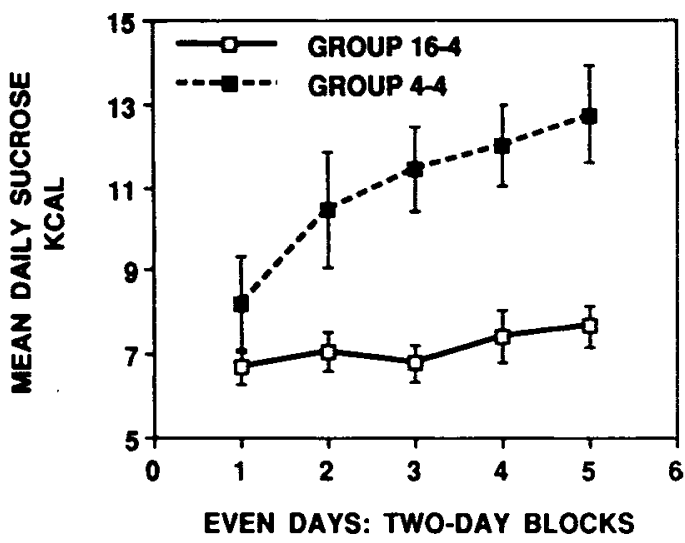

Figure 2. Mean daily sucrose intake (in kilocalories) over blocks of 2 even days in Experiment 1, as a function of the concentration of sucrose available on odd days. On odd days, Group 16-4 had 2-h access to $16 \%$ sucrose, while Group 4-4 had 2-h access to $4 \%$ sucrose. On even days, shown in the figure, both groups had 2-h access to $4 \%$ sucrose. Vertical lines indicate 1 SEM.

magnitude of the negative contrast effect remained fairly constant over days. A mixed ANOVA revealed a significant groups effect, reflecting the overall greater Purina intake of Group 4-4 [F(1,12) $=5.60, p<.05]$; a significant blocks effect, reflecting a general increase in Purina consumption over days $[F(4,48)=13.02, p<.001]$; but no significant interaction between groups and blocks $[F(4,48)=0.98]$.

Body-weight changes over Days 11-31. Figure 4 shows, for both groups, the mean change in body weight (in grams) over the 24-h interweighing intervals (1) between odd and even days and (2) between even and odd days, over Days 11-31. It will be recalled that the weighings occurred prior to the presentation of sucrose and $\mathrm{Pu}$ rina each day. Thus, differences in body weight between the two groups do not simply reflect differences in the immediate postmeal state of the animals. It can be seen in Figure 4 that during the 24-h interval between odd and even days, Group 16-4 gained significantly more body weight (5.5 vs. $0.8 \mathrm{~g})$ than did Group $4-4[t(12)=8.18$, $p<.001]$. However, during the interval between even and odd days, Group 16-4 lost weight while Group 4-4 gained weight $(-5.2$ vs. $0.2 \mathrm{~g}$ ), a difference that was significant $[t(12)=5.48, p<.001]$. Thus, if Group 16-4's caloric intake had merely kept pace with that of Group 4-4 on $4 \%$ days, Group 16-4 would have made substantial progress towards regaining their ad-lib level of weight over the course of Days 11-31, because of their weight gains on $16 \%$ days. Instead, however, the contrast-induced suppression of both sucrose and Purina consumption shown by Group 16-4 on $4 \%$ days caused them to lose the gains they had made on $16 \%$ days and, indeed, to lag behind Group 4-4, in terms of number of grams of body weight gained between Days 11 and 31 (2.9 vs. $10.2 \mathrm{~g}$ ), although that difference was not significant $[t(12)=0.86]$.

In summary: On days when the $4 \%$ sucrose solution was available, Group 16-4 consumed 17\% fewer total calories than they "should have," when the caloric intake of the unshifted control animals is used as a yardstick. That caloric shortfall reflects the consequences of two separate contrast effects. First, the shifted animals consumed significantly fewer sucrose calories when the $4 \%$ sucrose solution was available. This is the familiar NCCE, but obtained with relatively lengthy exposures to the sucrose solutions-a procedure that allows a calorically costly NCCE to emerge. Second, and more surprising, the shifted animals ate significantly less Purina Chow than did the control animals. This suppressed intake of the familiar maintenance diet occurred only on $4 \%$ days. On $16 \%$ days, Group 16-4 ate just as much Purina Chow as did Group 4-4. Thus, for animals that had experience with the $16 \%$ solution, the $4 \%$ solution engendered NCCEs, both to itself, and to a completely different source of calories. These negative contrast effects did not diminish

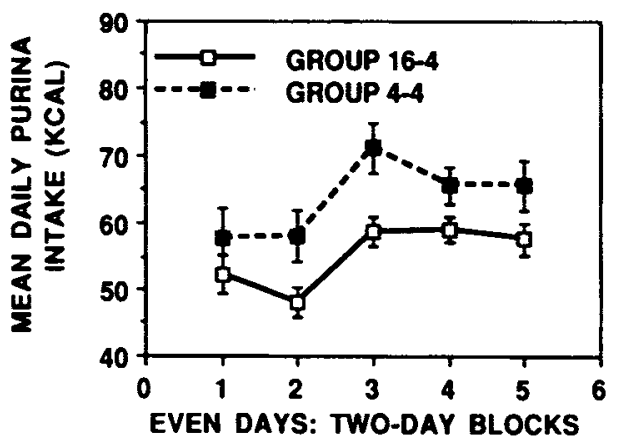

Figure 3. Mean daily Purina intake (in kilocalories) over blocks of $\mathbf{2}$ even days in Experiment 1, as a function of the concentration of sucrose available prior to the Purina meal on odd days. On odd days, Group $16-4$ had access to $16 \%$ sucrose prior to the Purina meal, while Group 4-4 had access to $4 \%$ sucrose prior to the Purina meal. On even days, shown in the figure, both groups had access to $4 \%$ sucrose prior to the Purina meal. Vertical lines indicate 1 SEM.

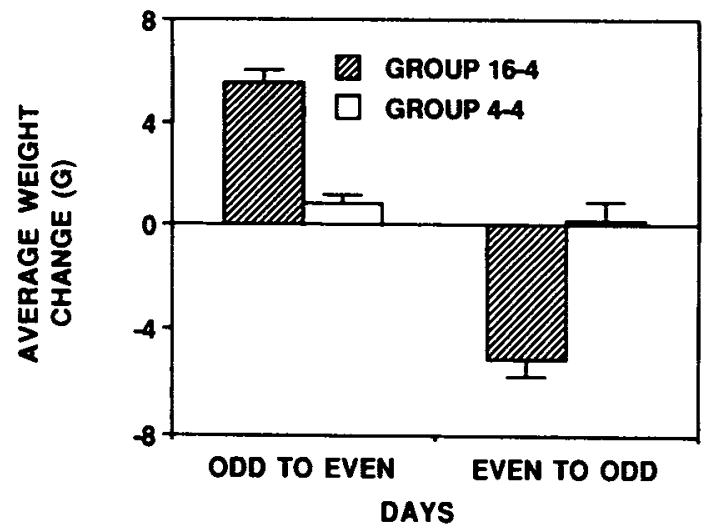

Figure 4. Average daily change in body weight (in grams) in Experiment 1 , as a function of the concentration of sucrose that was available for $2 \mathrm{~h}$, immediately prior to the 2-h Purina meal. On odd days, Group 16-4 had access to $16 \%$ sucrose, while Group $4-4$ had access to $4 \%$ sucrose. On even days, both groups had access to $4 \%$ sucrose. Vertical lines indicate 1 SEM. 
as a function of repeated experiences with the two different sucrose solutions for Group 16-4, and, indeed, in the case of sucrose intake, the size of the negative contrast effect increased as a function of repeated experiences with the $4 \%$ solution. These findings suggest that the negative contrast effect involving sucrose ingestion was not due to a neophobic reaction to the $4 \%$ solution (see Flaherty, 1982).

The second experiment was designed to explore two questions regarding the unexpected suppression of solidfood consumption caused by exposure of Group 16-4 to the $4 \%$ sucrose solution. First, will a 30 -min interval interposed between sucrose consumption and Purina consumption eliminate the NCCE with Purina Chow? Second, will a 5 -min exposure to the $16 \%$ solution, $25 \mathrm{~min}$ after exposure to the $4 \%$ solution, eliminate the NCCE with Purina Chow?

\section{EXPERIMENT 2}

\section{Method}

Subjects. The subjects were the animals used in Experiment 1, kept in the same groups.

Procedure. Following the completion of Experiment 1, the animals were placed on ad-lib food and water for 3 weeks, at the end of which their average weight was $598 \mathrm{~g}$. Then they were again placed on the 2-h/day Purina Chow schedule used in the first part of Experiment 1, for 10 days. On Day 11 and thereafter, the animals received exposure to sucrose solution(s) beginning $2 \mathrm{~h}$ before the Purina meal. Their average body weight on Day 11 was $513 \mathrm{~g}$.

The schedule of sucrose presentation varied across a 4-day cycle, which was repeated 3 times. On the " $A$ " day of each cycle, Group $16-4$ received a 90 -min exposure to the $16 \%$ sucrose solution, followed by a 25 -min no-solution (or water) interval, followed by a final 5 -min exposure to the $16 \%$ solution again, followed by 2-h access to Purina Chow and water. For Group 4-4, the $4 \%$ solution was presented in the same manner, prior to the Purina meal. On the "B" day of each cycle, Group 16-4 received a 90-min exposure to the $4 \%$ solution, followed by a 25 -min no-solution interval, followed by a final 5 -min exposure to the $16 \%$ solution, followed by the Purina meal with water. For Group 4-4, the $4 \%$ solution was presented both times, prior to the Purina meal. On the " $C$ " day of each cycle, the same schedule as on "A" days was in effect for both groups. On the " $D$ " day of each cycle, both groups received a 90 -min exposure to the $4 \%$ solution, followed by a 30 -min no-solution interval, followed by the Purina meal with water. Following the Purina meal on all days, water remained available until sucrose presentation the following day.

In short, on the 1st and 3rd day of each 4-day cycle, Group 16-4 received two exposures to the $16 \%$ solution, while Group 4-4 received two exposures to the $4 \%$ solution, prior to the daily Purina meal. On the second day, Group 16-4 received an initial exposure to the $4 \%$ solution, followed 25 min later by a subsequent exposure to the $16 \%$ solution, just prior to the daily meal, while Group $4-4$ received two $4 \%$ exposures (i.e., same sequence as on the 1 st and 3 rd days). On the 4th day of each cycle, both groups received exposure only to the $4 \%$ solution, but 30 min elapsed between the end of the sucrose exposure and the opportunity to eat Purina Chow. The questions of primary interest were: (1) Will the 30-min delay between drinking $4 \%$ sucrose and eating food eliminate the contrast effect with Purina Chow for Group 16-4 on " $D$ " days; and (2) will a brief exposure to the $16 \%$ solution, following exposure to the $4 \%$ solution, eliminate the contrast effect with Purina Chow for Group 16-4 on " $B$ " days?

\section{Results and Discussion}

" $A$ " and " $C$ " days. Figure 5 shows the average daily intake of Purina Chow (in kilocalories) for the two groups on (1) " $A$ " and " $C$ " days combined, (2) " $D$ " days, and (3) " $B$ " days, collapsed over the three repetitions of the 4-day cycle. It can be seen that on " $A$ " and " $C$ " days, when Group 16-4 had access to the $16 \%$ sucrose solution for a total of 95 min prior to the opportunity to eat Purina Chow, while Group 4-4 had similar access to the 4\% solution, Group 16-4 ate less Purina Chow than did Group 4-4, but the difference was not significant $[t(12)=1.18]$. However, Group 16-4 consumed more sucrose calories than did Group 4-4 on such days, 25.5 and $11.3 \mathrm{kcal}$, respectively; and that difference was significant $[t(12)=$ $8.34, p<.001]$. Thus, giving Group $16-4$ access to the $16 \%$ solution on " $A$ " and " $C$ " days in the second experiment resulted in the same general pattern of results as was found on $16 \%$ (odd) days in the first experiment: Group 16-4 ate significantly more sucrose kilocalories and fewer, but not significantly so, Purina kilocalories than did Group 4-4. And, as was the case on odd days in the first experiment, in Experiment 2, Group 16-4 gained significantly more weight following " $A$ " and " $C$ " days than did Group 4-4 $[t(12)=3.35, p<.01]$.

"D" days. On " $D$ " days, when all animals had a 30-min interval separating exposure to the $4 \%$ solution from the Purina meal, Group 16-4 ate an average of 57.6 Purina $\mathrm{kcal}$ and Group 4-4 ate an average of 64.1 Purina kcal, a difference that was not significant $[t(12)=1.75]$. There was a significant difference in sucrose intake on " $D$ " days, however, with Group 16-4 consuming an average of $6.8 \mathrm{kcal}$ and Group $4-4$ consuming an average of

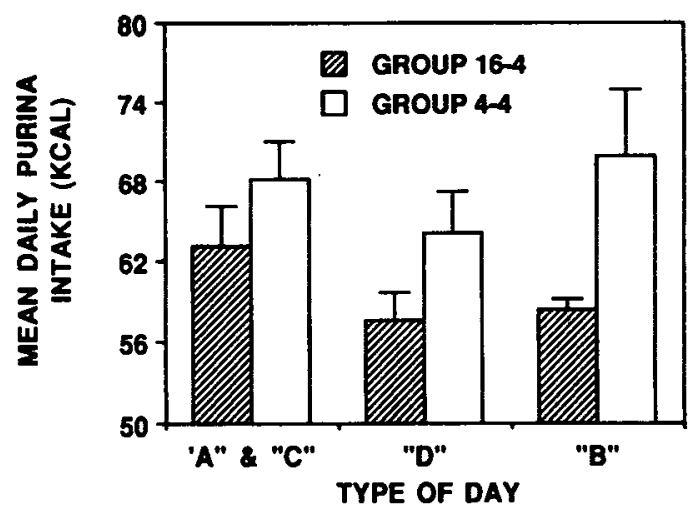

Figure 5. Mean daily Purina intake (in kilocalories) in Experiment 2, as a function of the concentration of sucrose solution(s) available prior to the Purina meal. On the "A" and " $\mathrm{C}$ " days of each 4-day cycle, Group 16-4 had 90-min access to $16 \%$ sucrose followed, 25 min later, by 5 -min additional access to $16 \%$ sucrose. Group $4-4$ had access to $4 \%$ sucrose during both the 90 - and 5-min intervals on " $A$ " and " $C$ " days. On the "B" day of each cycle, Group 16-4 had 90-min access to $4 \%$ sucrose followed, 25 min later, by 5-min access to $16 \%$ sucrose. Group $4-4$ had access to $4 \%$ sucrose both times. On the "D" day of each cycle, both groups had 90 -min access to $4 \%$ sucrose, followed $30 \mathrm{~min}$ later by the Purina meal. The 4-day cycle was repeated three times, and the data are collapsed over cycles. Vertical lines indicate 1 SEM. 
$10.8 \mathrm{kcal}[t(12)=4.03, p<.01]$. Thus, on " $\mathrm{D}$ " days, a significant negative contrast effect was obtained for sucrose intake but not for Purina intake. From this pattern of results, it might reasonably be concluded that, on " $D$ " days, the negative contrast effect involving Purina Chow was eliminated by the 30 -min delay between sucrose ingestion and Purina ingestion. However, it will be suggested below that the lack of a significant difference in Purina intake between the two groups on " $D$ " days reflects atypical data from one control animal, rather than a weakening of the NCCE in the experimental animals. It might be noted, in this regard, that Group 16-4 lost significantly more body weight on " $D$ " days than did Group 4-4 $[t(12)=2.27, p<.05]$, the same type of results that was found following even days in Experiment 1 .

"B" days. It can be seen in Figure 5 that the biggest difference in Purina intake in Experiment 2 occurred on " $B$ " days, with Group 16-4 consuming an average of 58.2 Purina kcal and Group 4-4 consuming an average of $69.9 \mathrm{kcal}$, a difference that was significant $[t(12)=$ $2.28, p<.05$ ]. On " $B$ " days, it will be recalled, Group 16-4 received a 90 -min exposure to $4 \%$ sucrose followed, $25 \mathrm{~min}$ later, by a 5 -min exposure to $16 \%$ sucrose; Group 4-4 received 90- and 5-min exposures to 4\% sucrose on " $B$ " days. When the significant difference in Purina intake for " $B$ " days is contrasted with the lack of such a significant difference for " $D$ " days, it suggests that Group 16-4's brief exposure to $16 \%$ sucrose on " $B$ " days strengthened, rather than weakened, the negative contrast effect produced by the 90 -min exposure to $4 \%$ sucrose. However, closer inspection of the data reveals that the different patterns of results for " $B$ " and " $D$ " days stem from differences in Group 4-4's Purina intake, rather than from changes in Group 16-4's intake. That is to say, whereas Group 16-4 ate nearly identical amounts of Purina on "D" and " $B$ " days $(57.6$ and $58.2 \mathrm{kcal}$, respectively), Group 4-4 ate less Purina on " $D$ " days $(64.1 \mathrm{kcal})$ than on " $B$ " days $(69.9 \mathrm{kcal})$. And, the drop in Purina intake on " $D$ " days for Group 4-4 was due, primarily, to a substantial and atypical change in the $\mathrm{Pu}$ rina intake of just one animal, which ate an average of $37.1 \mathrm{kcal}$ less on " $D$ " days than on " $B$ " days. For the remaining 6 animals in the group, the average " $B$ " minus " $D$ " difference in Purina intake was $0.6 \mathrm{kcal}$, with a standard deviation of $5.8 \mathrm{kcal}$. If the atypical data are removed from the analysis, it is then found that Group 16-4 ate significantly less Purina Chow on " $D$ " days than did the remaining 6 animals in Group 4-4 [t(11) $=2.58, p<.05]$.

Two additional points should be mentioned regarding the results of " $B$ " days. First, during the 90-min exposure to $4 \%$ sucrose, Group $16-4$ consumed significantly fewer sucrose kilocalories than did Group 4-4 $[t(12)=3.34$, $p<.01]$. Second, all of the animals in Group 16-4 consumed reasonable amounts of the $16 \%$ solution when it was made available: the average intake was $14.7 \mathrm{~g}$ $(=8.5 \mathrm{kcal})$, with a standard deviation of $1.8 \mathrm{~g}$. Thus, there was a significant NCCE to the $4 \%$ solution on " $B$ " days, and, there was a significant NCCE to Purina Chow as well, even though ingestion of the $16 \%$ solution intervened between ingestion of the $4 \%$ solution and ingestion of the Purina Chow. Again, it should be emphasized that the intake of $16 \%$ sucrose by itself (on " $A$ " and " $C$ " days) did not produce an NCCE to Purina Chow.

Therefore, the results of Experiment 2 suggest, provisionally, negative answers for both of the questions asked at the beginning of the experiment: It appears that neither (1) a 30-min delay by itself, nor (2) a 25-min delay plus a 5-min exposure to $16 \%$ sucrose, eliminates the NCCE to Purina Chow produced by exposure to $4 \%$ sucrose for Group 16-4.

\section{GENERAL DISCUSSION}

Previous research in the area of consummatory contrast has shown that when animals receive brief exposures to both a more and a less preferred incentive, their ingestion of the latter is strongly suppressed, relative to that of control animals that receive exposures only to the less preferred incentive (see Flaherty, 1982). Because of the brevity of the tests, however, such negative consummatory contrast effects (NCCEs) have not resulted in sizeable reductions in total daily caloric intake, and hence they have not presented a threat to the animals' physiological fitness. In the present research, the range of circumstances under which NCCEs can be obtained has been extended, with the consequent discovery that NCCEs can produce substantial reductions in total daily caloric intake, reductions that have a demonstrable effect on the ability of the animals to maintain body-weight levels.

In the first experiment, it was found that animals receiving alternating, daily exposures to $16 \%$ and $4 \%$ sucrose solutions for $2 \mathrm{~h}$ prior to their daily opportunity to eat Purina Chow (Group 16-4) showed an NCCE to the 4\% solution that persisted for at least 10 such exposures. The NCCE to the $4 \%$ solution cost these experimental animals approximately $4 \mathrm{kcal}$ on each occasion, compared to unshifted control animals that were always exposed to the $4 \%$ solution (Group 4-4). The experimental animals also showed a persistent NCCE of approximately $8.5 \mathrm{kcal}$ to the Purina Chow that they consumed during the 2-h meal following exposure to the $4 \%$ solution, with the consequence that they consumed $17 \%$ fewer total calories (sucrose + Purina) on days when they were exposed to the $4 \%$ sucrose solution than did the control animals. That $17 \%$ shortfall in caloric intake resulted in significant losses in body weight on $4 \%$ days for Group $16-4$, relative to Group $4-4$. Since both groups were at least $14 \%$ below their ad-lib weight levels throughout the series of tests, the weight loss exhibited by Group $16-4$ on $4 \%$ days must be viewed as potentially maladaptive.

It should be noted that the NCCE involving Purina Chow occurred only on 4\% days for Group 16-4. Purina intake was equivalent for the two groups on days when 
the experimental animals were given the $16 \%$ solution prior to the Purina meal. And, because of the caloric bonus provided by the $16 \%$ solution, the experimental animals actually consumed significantly more calories, and gained significantly more weight, than did the control animals on days when the experimental animals were given the $16 \%$ solution. Thus, if Group 16-4 had merely kept pace with Group 4-4 on 4\% days, Group 16-4 could have made substantial progress toward regaining their ad-lib level of weight over the course of the experiment.

The second experiment was designed to investigate whether the NCCE to Purina Chow, produced by exposure to the $4 \%$ solution for the experimental animals, would be eliminated (1) if a 30-min delay were interposed between exposure to the $4 \%$ solution and the opportunity to eat Purina Chow, or (2) if a 25-min delay plus a 5-min exposure to the $16 \%$ solution were interposed between exposure to the $4 \%$ solution and the opportunity to eat Purina Chow. The results showed that the NCCE to Purina Chow was still present under both conditions, although the effect was obscured in the 30-min-delay condition by atypical performance from one control animal.

Presumably, if enough time were allowed to elapse between exposure to the $4 \%$ solution and the start of the Purina meal, and/or if enough exposure to the $16 \%$ solution were inserted following exposure to the $4 \%$ solution, the NCCE for the maintenance diet would be eliminated in the experimental animals. These questions await resolution through additional research.

Upon completion of the present experiments, it was learned that in two previous studies contrast effects were obtained under conditions of lengthy exposure to the incentives. Rogers (1985) found that rats fed a highly palatable high-fat diet under ad-lib conditions for several days ate less of their standard maintenance diet, when returned to it under ad-lib conditions, than did control animals that were maintained on the standard diet. And Johnson, Ackroff, Peters, and Collier (1986) found that when nondeprived rats were taken, over consecutive days, through a series of diets that differed in caloric density, the animals showed inappropriate changes in the size of their initial meals, following a switch in nutrients. That is to say, following a change to a less calorically dense diet (which ought to be met by an increase in meal size, if caloric intake is to remain constant), the animals showed a decrease in the size of the early meals-an "inappropriate" change that could be taken as evidence of a contrast effect.
The results of the Johnson et al. (1986) and Rogers (1985) studies show that consummatory contrast effects can be obtained under ad-lib feeding conditions with nondeprived animals. The results of the present experiments show that, even when rats are substantially below their ad-lib weight levels, the manipulation of incentives during relatively lengthy meals can lead to persistent and stable contrast effects that are of such a magnitude as to lead to additional, significant losses in body weight. It would be interesting to know, in this context, whether the $4 \%$ solution would still lead to a reduction in body weight every time it was encountered, even if there were 2 or more $4 \%$ days for every $16 \%$ day. Similarly, it would be interesting to know whether the type of results obtained in the present study are dependent upon the regular alternation of incentives that was used, or whether an irregular sequence would produce the same effects. In any event, the present results indicate that, while the psychological mechanisms that underlie NCCEs may be of intrinsic interest to psychologists, the potential consequences of NCCEs on the organism's fitness may be of potential interest to physiologists and ecologists. In a wider context, NCCEs might be viewed as another example of the ways in which ecological variables-in this case, the variety of nutrients encountered-modulate the expression of "homeostatic"' mechanisms (see, e.g., Collier, 1983).

\section{REFERENCES}

Bolles, R. C. (1975). Theory of motivation. New York: Harper \& Row. Collier, G. H. (1983). Life in a closed economy: The ecology of learning and motivation. In M. D. Zeiler \& P. Harzem (Eds.), Advances in analysis of behaviour: Vol. 3. Biological factors in learning (pp. 223-274). Chichester, U.K.: Wiley.

FLAHERTY, C. F. (1982). Incentive contrast: A review of behavioral changes following shifts in reward. Animal Learning \& Behavior, 10 , 409-440.

Flaherty, C. F., Becker, H. C., \& Pohorecky, L. (1985). Correlation of corticosterone elevation and negative contrast varies as a function of postshift day. Animal Learning \& Behavior, 13, 309-314.

Johnson, D. F., Ackroff, K., Peters, J., \& Collier, G. H. (1986). Changes in rats' meal patterns as a function of the caloric density of the diet. Physiology \& Behavior, 36, 929-936.

Rogers, P. J. (1985). Returning "cafeteria-fed"' rats to a chow diet: Negative contrast and effects of obesity on feeding behaviour. Physiology \& Behavior, 35, 493-499.

(Manuscript received September 25, 1989; revision accepted for publication June 18,1990 .) 